\title{
PRESENTATION
}

\section{Catherine TAUVERON}

CELLAM Université Rennes 2 ctauveron@orange.fr

Au-delà de ses nombreuses énigmes (Qui est, par exemple, cet Henri-de-fer surgi du néant à la toute fin du conte et que le titre met en évidence en le plaçant au même rang que le Roi-grenouille ? Comment comprendre le comportement du père et de sa fille, de la fille et de la grenouille et, au-delà, ce qui se joue entre eux ? Pourquoi la mère est-elle absente ? Comment celle qui nous est présentée d'abord comme une toute petite fille peut-elle devenir en une nuit fille à marier à un inconnu ?...), « Le Roi-grenouille ou Henri-de-fer» des Grimm est le conte des paradoxes:

- Considéré par les Grimm comme « le plus ancien et le plus beau» des contes, il est placé en position inaugurale dès la première édition des Kinder- und Hausmärchen (1812) et ne changera pas de place tout au long des rééditions. Il est l'objet, de la part des auteurs, de nombreuses réécritures. Et l'on peut se demander si une telle position dans le recueil et le soin littéraire dont il a fait l'objet ne le rendent pas emblématique de l'ensemble du projet éditorial des auteurs. Cependant, en France du moins, il n'a fait l'objet d'aucune étude critique ample et approfondie.

- Sa dimension sexuelle (et agressive) est évidente, bien que les Grimm se soient employés à la gommer au fil des versions, si évidente que bon nombre de traducteurs historiques (il conviendrait plutôt de parler d'adaptateurs) prennent soin d'effacer ce que la scène du lit peut avoir de troublant 


\section{Catherine Tauveron}

et qu'à l'inverse l'on compte un nombre important de réécritures érotiques pour adultes, aux Etats-Unis notamment. Pour autant, les programmes français pour l'école en recommandent la lecture au cycle 2 (5-6-7 ans), jugée de fait adaptée aux préoccupations de la petite enfance.

- Il est certes toujours possible de lire le conte innocemment comme un conte didactique montrant qu'il est important et gratifiant à terme de tenir ses promesses... et d'obéir au père. Mais c'est oublier que la demande du père, imposant à la fillette une conjonction rebutante avec la grenouille, a quelque chose de scandaleux, que la capricieuse (et, à bien des égards, insupportable) fillette, si elle s’y était conformé, n'y aurait gagné qu'une sanction, le dégoût de nuits passées au contact d'une peau animale froide et visqueuse. En projetant la grenouille contre le mur, elle commet un acte d'une violence inouïe mais, ce faisant, autre paradoxe à surmonter, c'est cet acte violent de désobéissance qui ouvre la voie de la « récompense ». Pour reprendre Maria Tatar $^{1}$, les protagonistes des contes atteignent rarement leurs objectifs en observant des codes déontologiques stricts mais en l'occurrence :

\footnotetext{
Although the princess [...] is selfish, greedy, ungrateful, and cruel, in the end she does as well for herself as all the modest, obedient, magnanimous, and compassionate Beauties of 'The Search for the Lost Husband'. Much as the Grimms tried to rewrite the tale with paternal prompts about the importance of keepind promises and showing gratitude, they could not succeed in camouflaging the way in which the tale rewards indignant rage.
}

- Conséquence ou non de ce qui précède, le conte est relativement peu réédité en singleton dans sa version originale mais est la source d'innombrables réécritures. C'est que, sans avoir bénéficié, comme « Blanche-Neige », du vecteur disneysien, «Le Roi-grenouille ou Henri-de-fer» a cependant tout l'air de faire partie de la culture commune. Mais, s'il est indubitablement inscrit dans la mémoire collective, il s'avère qu'il est le plus mal connu des contes « connus ». Le baiser final accordé par la jeune fille à la grenouille (et l'heureuse transformation qui s'en suit) paraît à tout un chacun un fait établi, alors même qu'il ne figure pas dans le conte. Ce « souvenir induit » est partagé par le plus grand nombre des «réécriveurs», qui s’ « entre-réécrivent ». Et, du conte, ne reste, le plus souvent, que ce qui ne s'y trouve pas: la scène fantasmée et hollywoodienne du baiser, dont l'origine reste obscure. Ont disparu le puits et la balle, le père régisseur de la sexualité de sa fille, le dégoût et la rébellion violente de la jeune fille contre la proposition indécente de la

1 Maria Tatar, Off With Their Heads, Princeton, Princeton University Press, 1992, p. 154. 
grenouille, et pour finir, corps et biens, le fidèle Henri et ses cerceaux de fer.

Le présent numéro se donne pour objectif d'approfondir énigmes et paradoxes du conte. Il offre des éléments pour comprendre sa gestation et sa portée symbolique ainsi que son devenir éditorial et mémoriel au travers de l'étude de quelques-unes de ses traductions ou adaptations (en Europe et hors de sa sphère culturelle d'origine), de ses illustrations qui à elles seules valent interprétations et de ses réécritures multiformes à destination ou non de la jeunesse. Dans ces trois derniers cas de figure, plusieurs questions se posent auxquelles l'on tente de répondre: quel degré de fidélité au contesource, quelles reconfigurations, altérations, réorientations ou renforcements éventuels de la portée symbolique du conte-source ? Pour finir, le numéro s'intéresse à la réception du conte dans le monde de l'école qui en reste le principal vecteur, soit à la lecture spontanée de jeunes enfants saisie dans le cadre de la classe et à la lecture suggérée aux enseignants par les documents pédagogiques disponibles. 